\title{
MicroRNAs as emerging diagnostic and prognostic marker in cancer biology and therapy
}

\author{
Asif Khurshid Qazi, Ph.D., ${ }^{1}$ Fizalah Kawoosa, M.Sc., ${ }^{2}$ Zafar Amin Shah, Ph.D., ${ }^{3}$ \\ 'Senior Resident, ${ }^{2}$ Ph.D. Scholar, ${ }^{3}$ Prof. \& Head, Dept. of Immunology and Molecular Medicine, \\ SKIMS, Soura, Srinagar
}

\section{A B S T R A C T}

\begin{abstract}
MicroRNAs (miRNAs) contribute to cellular homeostasis and differentiation in many ways, and therefore play an important role in many pathophysiological events. The knowledge of small non-coding RNA molecules has developed our understanding towards basic processes of cancer biology and the molecular mechanisms underlying tumor initiation and progression. MiRNA research field has grown to be more and more attractive as evidence is emerging that miRNAs possibly play vital regulatory roles in all fundamental biological processes. Notably, as research continues to reveal the mechanisms underlying cancer therapy efficacy, it is apparent that miRNAs contribute to responses to drug therapy and are themselves modified by drug therapy. One important field for miRNA research is to identify functions of miRNAs and the associated signaling pathways in the initiation, progression, metastasis and drug-resistance of tumors in order to propose novel, efficient target based therapeutics that directly target pathologically essential miRNAs and their allied target genes. Currently, miRNA signatures have increasing importance and use in the diagnosis and prognosis of various types of cancers. In this review, we have highlighted the role of various miRNAs in various cancers and other disease so that future of miRNA research will provide more interesting outcomes in terms of therapy and cure. JMS 2016; 19(2):47-58
\end{abstract}

Keywords: miRNAs; Cancer biology; Oncogene; Tumor suppressor; Drug therapy.

\section{INTRODUCTION}

MicroRNAs (miRNAs) are small non-coding RNAs that vary in size from 19 to 25 nucleotides. They are endogenous, stable, conserved and play an important role by targeting mRNAs for translational suppression or degradation. MiRNAs were first discovered in 90s, since then at least two thousand human miRNAs have been identified and classified, which have been proven to play an essential part in various biological processes i.e. cell proliferation, cell cycle progression, apoptosis and differentiation'. Additionally, microRNAs include one of the most abundant classes of gene regulatory molecules in multicellular organisms and probably influence the output of many genes encoding proteins. Furthermore, promising confirmation suggested a wide range of essential cellular processes such as cell proliferation, progression, growth,

\section{*Correspondence:}

1. Dr. Zafar Amin Shah Professor \& Head

Dept. of Immunology \& Molecular Medicine, SKIMS,

E-mail: zaffaramin@gmail.com

2. Dr. Asif Khurshid Qazi

Dept. of Immunology \& Molecular Medicine, SKIMS,

E-mail: asifkqazi@gmail.com mobility, metastasis and apoptosis, as well as carcinogenesis. The expression patterns of microRNAs exhibited a relationship with characteristic clinicopathological parameters in cancer subtypes thereby highlighting microRNAs as promising diagnostic and prognostic biomarkers for different human cancers. Moreover, in various cancers, large number of miRNAs have been identified and they are known to be involved in modulating cancer cell characteristics and behavior ${ }^{2}$. It has been observed that miRNAs dysregulation is responsible for the pathogenesis of majority of cancers starting from initiation to metastatic phase ${ }^{3}$. Moreover, circulating miRNAs are reproducible and stable in the body fluids of many cancer patients, and are deregulated in pancreatic, breast, colon, leukaemia and lung cancer ${ }^{4}$. Notably, the role of miRNAs in the development and overall regulation of tumors shows great promise to screen therapy targets. Despite the fact that molecular targeted therapies to various cancers have shown potential results, no promising targeted therapy has been useful for a large number of cancer patients. Although, there has been improvement in early diagnosis, most cancers are still diagnosed at the last stage. Hence the recognition of novel diagnostic biomarkers or treatment strategies is critical and indispensable for the 
control of cancer.

\section{Role of MiRNA in Cancer}

\section{Colorectal cancer}

Currently, miRNAs have been shown to be promising sensitive and specific early detecting molecular biomarkers. There are reports that plasma levels of miR-29a and miR-92 (Table 1) are significantly increased whereas levels of miR601 and miR-760 are significantly decreased in the early stage of CRC as compared to controls ${ }^{5}$. Recently, it was reported that in CRC, four cell-free miRNAs i.e. miR-31, miR-181b, miR-92, miR-203 (Table 1) are down-regulated and two miRNAs i.e. miR-21, let-7g (Table 1) are upregulated, these being more sensitive and specific in the identification of CRC than any single protein biomarker like carcino-embryonic antigen (CEA) or cancer antigen 19.9 (CA19-9) ${ }^{6}$. Moreover, it has been reported that a combination of seven exosomal miRNAs (let-7a, miR-23a, miR-21, miR-150, miR-223, and miR-1229) might be used for early detection of CRC, being significantly increased as compared to healthy groups. In addition, high levels of serum miR-200c (Table 1) in CRC patients have been reported to have a powerful interrelationship with lymph node metastasis ${ }^{7}$.

\section{Gastric cancer}

For Gastric cancer (GC) diagnosis, fewer numbers of sensitive and specific markers are available. Several studies reported that many extracellular miRNAs are promising and sensitive specific biomarkers for early diagnosis of $\mathrm{GC}^{34}$. More recently, it was reported that plasma levels of miR-18a (Table 1) are significantly increased in GC patients ${ }^{8}$. In addition, panel of five miRNAs (miR-16, miR-25, miR-92a, miR-451, and miR-486-5p) was identified in plasma as a potential biomarker for early diagnosis of $\mathrm{GC}^{35}$. Moreover, another study showed that the plasma miR- 122 was downregulated and plasma miR-192 was up-regulated in GC patients and might be used for the early diagnosis of distant metastasis $^{9}$ (Table 1).

\section{Breast cancer}

Breast cancer is a highly heterogeneous disease with different molecular alteration, which result in its classification into five intrinsic subtypes (luminal A and B, HER2-enriched, basal-like and normal-like) ${ }^{10}$. Recently, several studies reported that deregulation of miRNAs expression is strongly associated with molecular breast cancer subtype. In plasma, combination of four (miR-29a,
miR-181a, miR-223, miR-652) (Table 1) down-regulated and three (miR-29a, miR-181a, miR-652) up-regulated miRNAs has been shown to be significantly and reliably associated with luminal A tumor ${ }^{11}$. Similarly, tissue miRNA-342 has been found to be highly expressed in luminal B tumor and regarded as a very sensitive and specific biomarker in this type of breast cancer (64). Moreover, other miRNAs have been identified to distinguish the remaining subtypes, such as basal type (miR-18a, miR-135b, miR-93, and miR-155), HER2 type (miR-142-3p, and miR-150), and normal type (miR-145, miR-99a, miR-100, miR-130) ${ }^{10}$

\section{Pancreatic cancer}

Cancer of the pancreas is now recognized as one of the most important medical problems facing the male population. Notably, after initial reports indicating serum miR-141 to be very sensitive biomarker for $\mathrm{PCa}$, different and contradictory results were obtained ${ }^{12}$. Other studies have reported that extracellular miR-375 and miR-141 are most optimistic markers in the diagnosis of this neoplasm ${ }^{13}$. Urine, another important source of circulating miRNA, has been widely exploited for the diagnosis of urological cancer. It has been reported that miRs 107 and 574-3p were significantly higher in the urine of PCa patients compared to healthy groups, and would perform even better than PCA3 normalized to PSA in identifying the presence of PCa ${ }^{14}$. Recently, it has been reported that urine miR-205 and miR214 were significantly down-regulated in PCa patients with specificity of $80 \%$ and sensitivity of $89 \%{ }^{15}$.

\section{Lung cancer}

The lack of specific and sensitive tools for early diagnosis as well as inadequate targeted therapies contribute to poor outcomes of lung cancer. In lung cancer, a signature of five miRNAs has been used in the prediction of treatment outcome for non-small-cell lung carcinoma (NSCLC), among which high expression of miR-221 and let-7a is associated with good prognosis, as opposed to elevated miR-137, miR-372, and miR-182 associated with poor prognosis ${ }^{16}$. Likewise, high expression of the miR-183 family (miR-183, miR-182, and miR-96) has been reported to be associated with overall poor survival in patients with lung cancer ${ }^{17}$. Plasma miR-21, miR-145, and miR-155 (Table 1) used in combination helped in distinguishing lung cancer from healthy smokers with $69.4 \%$ sensitivity and $78.3 \%$ specificity ${ }^{18}$. In combination of miR-205, miR-210 
and miR-708 (Table 1) in sputum distinguished lung squamous cell carcinoma patients from normal subjects with $73 \%$ sensitivity and $96 \%$ specificity thus helping in the early detection of lung squamous cell carcinomas ${ }^{19}$. Furthermore, it has been found that high expression of serum miR-21 in lung cancer is associated with poor prognosis and the dynamic change of serum miR-21 between postoperative and preoperative lung carcinoma patients can potentially serve as biomarkers for disease recurrence after surgery ${ }^{20}$.

\section{Prostate cancer}

Prostate-specific antigen (PSA) that is currently available as a biomarker for prostate cancer, displays an inferior sensitivity and specificity. In prostate cancer, miRNAs such as miR-141 and miR-375 (Table 1) have been connected to prognosis of PC, which in turn correlate with high Gleason score or lymph-node positive status ${ }^{21}$. Studies have shown relationship of two miRNAs (miR-182 and miR-187) most differentially expressed in prostate cancer with the clinicopathological characteristics and outcome of patients, and it was observed that miR-187 expression was decreased in advanced prostate cancer cases ${ }^{22}$. In another study, a distinct set of 11 miRNAs was present at significantly greater amounts in prostate cancer patients with metastasis compared to those without metastasis. Moreover, diffrential expression profiles of miRNAs either downregulated or upregulated have been observed in prostate cancer ${ }^{23}$. It is also depicted that the tumor-associated miRNAs in serum (miR-26a, miR-32, miR-195, miR-let7i) allow noninvasive discrimination of prostate cancer $(\mathrm{PCa})$ and benign prostatic hyperplasia $(\mathrm{BPH}){ }^{24}$. Several reports indicate that circulating miR-26a-1 and miR-141 are associated with stage ${ }^{25}$, grade ${ }^{26}$ and cancer recurrence in patients with prostate cancer ${ }^{27}$.

\section{Hepatocellular carcinoma}

Current diagnostic methods available for Hepatocellular carcinoma (HCC) are insufficient for detecting the disease at early stages. Accurate, noninvasive biomarkers for the early detection of HCC are urgently needed to reduce worldwide morbidity and mortality related to HCC. MicroRNAs (miRNAs) have shown great promise as tissuebased markers for HCC diagnosis and prognosis. Moreover, they are stably expressed in serum and urine, and these circulating microRNAs (cmiRNAs) are emerging as novel noninvasive biomarkers for the early detection and prognosis of HCC. It has been reported that four miRNAs (miR-92, miR-20, miR-18 and precursor miR-18), were associated with the HCC differentiation status ${ }^{28}$. Furthermore, different subsets of miRNAs may be involved in the distinct biological characteristics of HCC. For example, epithelial cell adhesion molecule-positive human liver cancer stem cells are positively correlated with the expression of miR-181 ${ }^{29}$. A study that was based on the analysis of clinical sample data, identified a 20-miRNA metastasis signature that could predict primary HCC with venous metastases ${ }^{30}$. In addition, a number of studies have indicated that certain miRNAs are associated with distinctive liver tumor risk factors. These include the observation that miR-26a-1 expression exhibits gender differences ${ }^{31}$, that miR-96 is overexpressed in HBV-related $\mathrm{HCC}$, and that the downregulation of miR-126 is associated with alcohol use ${ }^{32}$. Previous studies have revealed that miR221/222, miR-21, miR-224 and miR-34a that are known to promote tumor progression are consistently upregulated in $\mathrm{HCC}^{32}$

\section{Esophageal cancer}

Esophageal cancer (EC) is one of the most common malignant tumors worldwide. The incidence and mortality of esophageal cancer (EC) are high, and rank eighth and sixth, respectively, out of all types of cancer, affecting more males than females ${ }^{33}$. Notably, aberrant expression of 46 miRNAs in EC tissues, of which 7 miRNAs (upregulation of miR-25, miR-424 and miR-151; downregulation of miR100, miR-99a, miR-29c and miR-140) can be used as biomarkers to distinguish malignant EC lesions from adjacent normal tissue. Moreover, miR-335, miR-181d, miR-25, miR-7 and miR-495 were associated with the general pathological type of EC; miR-25 and miR-130b were associated with the degree of differentiation of EC and the expression level of miR-103/107 was negatively correlated with the survival rate of EC patients and may be used for early diagnosis as well as gene therapy targets for $\mathrm{EC}^{34}$. Downregulation of miR-203 and miR-205 compared with normal epithelium in EC, while as overexpression of miR-21 (Table 1) in the two types of EC has been reported. Similarly, miR-200c, miR-194 and miR-192 have been shown to be downregulated in ESCC, but significantly upregulated in $\mathrm{EAC}^{35}$. 
TABLE 1 | Expression and source of miRNA in various human cancers

Cancer Type MiRNA Source Expression

Lung cancer

Breast cancer

Colorectal cancer

Prostate

Gastric cancer

Hepatocellular carcinoma

Cervical

Esophageal

Bladder

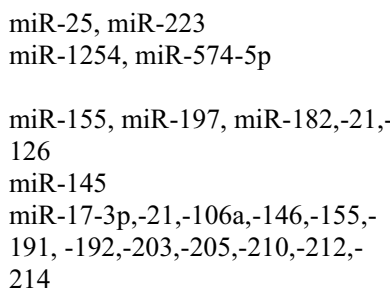

Serum Down

Serum Differential

Serum Up

Plasma Down

Urine Up

Urine Down

Plasma Up

Plasma Differential

Plasma Up

Serum Up

Serum Up
MiR-15b, miR-130b, and miR-16 Serum

miR-21,-1,-25,-206,let-7f

Serum

miR-625, miR-618, miR-532, miR-

650 ,

miR-516-5p

$\operatorname{miR}-218$

miR-21

miR-125b and mir-126

miR-129,-133b

miR-26-b-5p,-144-5p,-374-5p
Urine

$\begin{array}{ll}\text { Serum } & \text { Down } \\ \text { Serum } & \text { Up } \\ \text { Urine } & \text { Differential } \\ \text { Blood } & \text { Up } \\ \text { Blood } & \text { Up }\end{array}$


B-cell Lymphoma

T-cell Lymphoma

Leukemia

Pancreatic cancer

Renal cell carcinoma

Thyroid

Brain

Head and neck

Ovarian cancer
miR-21, miR-155 and $\mathrm{miR}-221$

miR-10393-3p

miR-10397-5p, NOVELMOO288M

miR-5586-5p

miR-30b

miR-515-3p, miR-155, miR-598, miR-

625

miR-199a-5p

miR-652, miR-627, miR-519e, miR$487 \mathrm{~b}$,

miR-124, miR-325, miR-181a, miR-

618

miR-512-3p, miR-886-5p, miR-886-

$3 \mathrm{p}$,

miR-708, miR-135b

miR-146a, miR-155

miR-210, miR-197, miR-191, miR-

$512-3 p$

miR-451, miR-146a, miR-22, miR-

455-3p,

miR-455-5p, miR-143, miR-494

miR-150, miR-342

miR-155

miR-181b-5p

miR-141, miR-375

miR-107, 574-3p

miR-195, miR-15b, miR-99a, miR-191

miR-200b

miR-211

miR-210

let-7f, miR-151-5p, miR-222

miR-15b*, miR-23a, miR-133a, miR-150*, miR-197, miR-497, miR-548b-5p

miR-125a, miR-200a

miR-205, miR-let-7f

miR-21,-92,-93,-126,-29a

\begin{tabular}{ll} 
different & Up \\
Tissue & Up \\
Tissue & Up \\
Tissue & Up \\
Tissue & Down \\
Tissue & Differential \\
& \\
Tissue & Differential \\
& \\
Tissue & Differential \\
Tissue & Up \\
Tissue & Down \\
Tissue & Up \\
Tissue & Down \\
Plasma & Up \\
Plasma & Up \\
\hline
\end{tabular}

Serum

Differential

Urine

Differential

Up

$\mathrm{Up}$

Up

Up

Up

Serum

Down

Saliva

Down

Plasma

Differential

Serum
Up 


\section{Bladder cancer}

Recently, numerous studies demonstrated that microRNAs are emerging as diagnostic biomarkers for bladder cancer. Specific miRNA profiles have been identified for several samples from patients with bladder cancer. In BC, 10 miRNAs has been found up-regulated ${ }^{36}$, the most commonly up-regulated miRNA in BC being miR-129 ${ }^{37}$. The authors found that the levels of miR-200 family members miR-141, miR-155, and miR-429 were lower in urine sediment in BC patients. Levels of miR-200a, miR200b, miR-200c, miR-141, miR-429, miR-205, miR-192, and miR-146a increased significantly after surgery. These findings suggest that miR-200-family miRNAs (miRs-141, $-429,-192,-146 \mathrm{a},-141$, and others) are promising as noninvasive, diagnostic, and prognostic markers. Also, studies have reported that miR-100, miR-125b, miR-130a, miR139-5p, miR-145, miR-199a-3p, miR-214 and miR-222 are down-regulated in invasive bladder tumors compared to superficial tumors, as well as in other tumor entities whereas expressions of miR-20a, miR-106b, miR-141, miR-130b, miR-200a, miR-200a and miR-205 were found to be upregulated in bladder cancer ${ }^{38}$.

\section{B-cell lymphomas}

B-cell lymphomas make up a heterogeneous group of lymphoproliferative disorders that originate from B cells and whose pathogenesis is still largely unknown. Accurate lymphoma diagnosis, prognosis and therapy still require additional markers. Diffuse large B-cell lymphoma (DLBCL) represents the most common lymphoid malignancy. DLBCL was one of the first lymphomas to be linked with aberrant miRNA expression, in particular the observation that miR-155 (Table 1) is highly expressed in this malignancy ${ }^{39}$. The levels of six miRNAs (miR-223, miR-217, miR-222, miR-221, let-7i and let-7b) have been found to be differentially expressed between cases of FL (follicular lymphoma) that underwent high grade histological transformation compared to those that did not.

\section{T-cell lymphomas}

T-cell lymphomas form a diverse group of T-cell derived lymphoid neoplasms comprising 10-15\% of non-Hodgkin's lymphomas. Increased expression of miR-122 in CTCL (Cutaneous $\mathrm{T}$ cell lymphoma) prevents chemotherapyinduced apoptosis. Therefore, targeting miR-122 may improve the outcome of chemotherapy ${ }^{40}$. Downregulation of miR-342 and upregulation of miR-17-5p were found to decrease and increase cell apoptosis in SzS respectively, which is involved in pathogenesis of the disease ${ }^{41}$. Upregulation of miR-21, miR-486, and miR-214 is involved in promoting cell survival in $\mathrm{SzS}$, and contributes to resistance to apoptosis in CTCL cell lines, and even may be a new diagnostic or prognostic biomarker for this type of incurable CTCL. MiR-155 (Table 1) is upregulated in MF ${ }^{42}$. Expression level of miR-21 and miR-155 is specifically higher in NK-cell lymphoma line than normal NK cells ${ }^{43}$.

\section{Renal cell carcinoma}

Renal cell carcinoma (RCC) is the most common malignant solid tumor in adults. It has been reported reported that there is upregulation of miR-28, $-185,-27$, and let-7f-2 in RCC compared to normal kidney ${ }^{44}$. Reports from other group showed that miR-34a, -224 and -21 were upregulated, whereas miR-141, -149 and -429 were downregulated in the clear cell RCC tissues ${ }^{45}$. Also it has been found that the expression of miR-21 was significantly upregulated in RCC compared with healthy kidney. In another study, miR-34a, 21 and -224 were upregulated, miR-141 was downregulated in the sera of patients with ccRCC, and the serum miR-21 expression levels were significantly correlated with the clinical staging of the patients with $\mathrm{ccRCC}{ }^{46}$. It was found that RCC patients presented higher circulating expression levels of miR-221 and -222 than healthy individuals ${ }^{47}$. MiRNA-based therapies (miR-381, miR-185, miR-141) may also be used together with other therapeutic strategies in pre-clinical studies ${ }^{48}$.

\section{Cervical Cancer}

Cervical cancer is a primary cancer of the uterine cervix in females and has a high incidence and mortality. miR-126 and miR-21 found in the serum have been found to be associated with cervical cancer ${ }^{49}$. Overexpression of miR21, miR-27a, miR-34, miR-34a, miR-146a, miR-155, miR196a, miR-203, and miR-221 has been detected in the serum, with miR-21, miR-27a, miR-34, miR-34a, and miR196 a being particularly highly expressed in squamous cell carcinoma of the cervix ${ }^{50}$. These results indicate that miRNA levels in serum can be used for diagnosis of cervical cancer and that some miRNAs may be useful for prediction of tissue type and for early diagnosis of cervical cancer. Several miRNAs in serum have been identified as candidate markers for lymph node metastasis in cervical cancer. A study conducted by researchers showed miRNA in patients with small cell carcinoma of the cervix (SCCC). Expression 
levels of 7 seven miRNAs, let-7c, miR-10b, miR-100, miR125b, miR-143, miR-145, and miR-199a-5p, were markedly suppressed in patients with advanced SCCC in comparison with those in patients with early SCCC. In patients with lymph node metastasis, expression of let-7c, miR-100, miR-125b, miR-143, miR-145, and miR-199a-5p was particularly suppressed ${ }^{51}$. Hypermethylation of miR149, miR-203, and miR-375 has also been found in HPVpositive high-grade dysplasia ${ }^{52}$, and miR-203 and miR-375 may be markers for precancerous conditions in the uterine cervix.

\section{Thyroid Cancer}

Thyroid cancer (TC) is a group of neoplastic disease arising from thyroid gland. Follicular cells of the gland give a rise for most of the TC (95\%) while small portion of cancers $(5 \%)$ is developing from para-follicular $\mathrm{C}$ cells and referenced as medullary TC. TC accounts for $95 \%$ of all endocrine malignancies and its incidence is constantly increasing ${ }^{53}$. The most consistently overexpressed miRNAs in well-differentiated TC types appear to be miR-221, miR222, and miR-146b ${ }^{54}$. Other commonly reported overexpressed miRNAs are miR- $155^{55}$ and miR-181 ${ }^{56}$. It has been reported that a set of four miRNAs (miR-146b, $221,-187,-30 d)$ could differentiate a malignant thyroid lesion from a benign tumour ${ }^{57}$. There are reports that the serum levels of let-7e, miR- 151 and miR-222 (Table 1) are significantly overexpressed in TC patients ${ }^{58}$. Another study has reported that $\mathrm{miR}-146 \mathrm{~b}$ and -155 can be used to discriminate between benign and malignant tumours ${ }^{59}$.

\section{Ovarian cancer}

Carbohydrate antigen-125 (CA-125) is the most frequently used biomarker for ovarian cancer detection ${ }^{60}$, but it is only elevated in approximately $50 \%$ of stage I EOCs and $70 \% 90 \%$ of advanced cases ${ }^{61}$. Hence, there is a clamant need for novel biomarkers that are more sensitive and specific for detecting OC when used alone or in combination with CA-125. Several microRNA signatures that are unique to ovarian cancer have been proposed, and serum-circulating microRNAs (miR-200a, miR-200b, miR-200cmiR-26a, miR-132, miR-145, and let-7b) have the potential to be useful diagnostic and prognostic biomarkers ${ }^{62}$. It has been reported that 4 miRNAs (miR141, miR-200a, miR-200b, and miR-200c) were upregulated and 25 out of 29 were downregulated, including miR-199a, miR-140, miR-145, and miR-125b-1 in the cancer samples when compared with normal tissues ${ }^{63}$. This study also found that miR signatures were different between ovarian carcinoma histotypes (serous, endometrioid, clear cell, and mucinous). It has also been stated that clear cell histotype is characterized by higher expression of miR-30a$5 \mathrm{p}$ and miR-30a-3p, whereas mucinous histotype displays higher levels of miR-192 and miR-194 ${ }^{64}$. There are reports suggesting that targeting miR-92a may prove to be a novel and effective gene therapy for patients with ovarian cancer ${ }^{65}$.

\section{Brain tumor}

Brain tumors are one of the top 10 causes of cancer deaths and a timely and accurate diagnosis is essential for disease management. Brain tumors could be classified into glial tumors (e.g. glioblastoma, astrocytomas, oligodendroglial tumors, ependymal tumors), embryonal tumors (e.g. medulloblastomas), tumors of the meninges (e.g. meningiomas), tumors of the hematopoietic system and tumor of the sellar region. Glioblastoma multiforme (GBM) is the most malignant and frequent brain tumor in adults, accounting for about $50 \%$ of diffuse gliomas ${ }^{66}$. Studies have reported strong upregulation of miR-221 in glioblastoma while downregulation of miR-128, miR-181a, miR-181b, and miR-181 ${ }^{67}$. Another study showed a downregulation of microRNA-124 and microRNA-137 in anaplastic astrocytoma and glioblastoma multiforme compared to normal brain and an upregulation of mir-21 ${ }^{68}$. High expression of miR-21 has been found to be significantly associated with poor patient survival, and in combination with other aberrant expressed microRNAs (low expression of miR-181b or miR-106a). MiR-21 profiling has potential application as novel diagnostic and prognostic indicator that may contribute to improve the selection of patients for adjuvant therapy and to predict the post-surgical outcome ${ }^{69}$. The miRNAs analysis in the serum of malignant astrocytoma patients, compared to normal controls, revealed several miRNAs (miR-15b, miR-23a, miR-133a, miR-150, miR-197, miR-497 and miR-548b-5p) (Table 1) whose concentrations were significantly lower in serum of the patients. Moreover, the concentrations of miR-23a, miR-150, miR-197 and miR-548b-5p were markedly decreased in malignant cases if compared to the astrogliosis group $^{70}$. Several miRNAs are up- (let-7a, let-7f, miR- 17, miR-21, miR-155, miR-17, miR-16, miR-26b, miR-374a, let-7d, miR-20a, miR-15b, miR-7b, miR-9) or down- 
regulated (miR-132, miR-134, miR-7, miR-330-3p, miR$127-3 p$ ) in oligodendrogliomas compared to non-neoplastic samples ${ }^{71}$. Some studies have identified 28 miRNAs (miR10a,miR-17-5p, miR-19a, miR-19b, miR-20a, miR- 21, miR-32, miR-34a, miR-106b, miR-130a, miR-135a, miR142-3p, miR-193a, miR-210, miR-301, miR-449b, miR502, miR-518b, miR- 551b, miR-565, miR-591, miR-594, miR-60miR-139, miR-323, miR-383, miR-433 and miR485- 5p) that were differentially expressed in ependymoma samples ${ }^{72}$. MiR-203 has been proposed as a biomarker for management of disease progression in ependymomas and as a predictor for the likelihood of relapse. Metastatic behavior of medulloblastoma has been reported to involve let-7 microRNAs ${ }^{73}$.

\section{Leukemia}

Studies have revealed that miRNAs may serve as biomarkers in clinical diagnosis of leukemia. It has been demonstrated that miR-128a, miR-128b, let-7b and miR223 are the most significant and discriminatory between AML and acute lymphoblastic leukemia (ALL) ${ }^{74}$. Further it has been confirmed that miR-128, let-7b, miR-223 and miR-181a have a diagnostic value in acute leukemia patients ${ }^{75}$. Another study demonstrated that the expression level of miR-142-3p and miR-29a in peripheral blood mononuclear cells (PBMNC) could be used as novel diagnostic marker in AML ${ }^{76}$. In CLL, several miRNAs including miR-181, miR-30d and let-7a that are differentially expressed between CLL lymphocytes and CD19+ control cells have been identified ${ }^{77}$. Downregulation of miR-326 has been reported in chronic myelogenous leukemia (CML) ${ }^{78}$. In ALL, miR-148, miR151 and miR-424 were identified as discriminative of Tlineage versus B-lineage ALL ${ }^{79}$. Accumulating evidence has shown that miRNAs can be used as biomarkers to predict the prognosis of patients. Specific extracellular miRNAs in body fluids and circulating miRNAs that have been found to be involved in leukemia include miR-92a, miR-638, miR-150, miR-342 and miR-181b-5p (191,192,193) (Table 1).

\section{Head and Neck cancer}

Head and neck cancer (HNC) is the sixth most common cancer in the world ${ }^{80}$, referring to cancers of the aero digestive tract, including lip, oral cavity, nasal cavity, paranasal sinuses, pharynx, larynx, oropharynx, hypopharynx, salivary glands, and local lymph nodes ${ }^{81}$. It has been suggested that specific miRNAs can be used as diagnostic markers for HNSCC (head and neck squamous cell carcinoma), such as overexpression of miR-21 and -205 ${ }^{82}$. It has been suggested that high expression of mir-21 can be used as an independent predictor of poor survival for patients with tonsillar SCC (squamous cell carcinoma) and a significantly lowered 5-year survival in patients with HNSCC ${ }^{83}$, while lowered miR-205 expression ${ }^{84}$ and increased miR-451 expression ${ }^{85}$ have been significantly correlated with locoregional relapse of HNSCC irrespective of disease severity at diagnosis and treatment. MiR-125a and -200a (Table 1) are two salivary miRs that have been shown to be markedly reduced in oral carcinoma patients versus healthy controls ${ }^{86}$. Moreover, plasma miRs have been shown to be reduced after tumor resection, implying that these miRs may be released from cancerous tissues into circulation and their potential use as a marker for disease progression. This was seen in plasma and salivary miR-31 ${ }^{87}$ and plasma miR-184 ${ }^{88}$.

\section{CONCLUSION}

In conclusion, miRNAs modulate the expression of target mRNAs in different pathways and themselves are also regulated by multiple factors. Based on increasing numbers of specific miRNA functional study, it is indispensable for us to construct a global view about miRNA regulation mechanisms and understand miRNA as diagnostic and prognostic marker.

\section{REFERENCES}

1. Kanellopoulou C. \& Monticelli S. A role for microRNAs in the development of the immune system and in the pathogenesis of cancer. Seminars in cancer biology 2008; 18: 7988.

2. Lovat F, Valeri $\mathrm{N} \&$ Croce CM. MicroRNAs in the pathogenesis of cancer. Seminars in oncology 2011;38:724733.

3. Dela CF \& Matushansky I. MicroRNAs in chromosomal translocation-associated solid tumors: learning from sarcomas. Discovery medicine 2011;12: 307317.

4. Iorio MV \& Croce CM. MicroRNA dysregulation in cancer: diagnostics, monitoring and therapeutics. A comprehensive review. EMBO molecular medicine 2012;4:143159.

5. Ayaz L GA. Yaroglu HY. Ozcan C. Tamer L. Differential expression of microRNAs in plasma of 
patients with laryngeal squamous cell carcinoma: potential early detection markers for laryngeal squamous cell carcinoma. $J$ Cancer Res ClinOncol 2013;139(9).

6. Wang J. Huang SK. Zhao M, Yang M. Zhong JL. Identification of a circulating micro RNAs nature for colorectal cancer detection. PLoSOne 2014;9.

7. Toiyama Y. Hur K. Tanaka K. Inoue Y. Kusunoki M. Boland CR. Serum miR-200 cis a novel prognostic and metastasis-predictive biomarker in patients with colorectal cancer. Ann Surg 2014 259(4):73543.

8 Tsujiura M. Komatsu S. I chikawa D. Shiozaki A. Konishi H. Takeshita H. Circulating miR-18a In plasma contributes to cancer detection and monitoring in patients with gastric cancer. Gastric Cancer 2014;18: 1 .

9. Chen Q. Ge X. Zhang Y. Xia H. Yuan D. Tang Q. Plasma miR-122 and miR-192 as potential novel biomarkers for the early detection of distant metastasis of gastric cancer. Oncol Rep 2014; 31(4):186370.

10. Blenkiron C. Goldstein LD. Thorne NP. Spiteri I. Chin SF. Dunning MJ. microRNA expression profiling of human breast cancer identifies new markers of tumor subtype. Genome Biol 2000; 8(10):R214.

11. McDermott AM. Miller N. Wall D. Martyn LM. Ball G. Sweeney K J. et al. Identification and validation of oncologic miRNA biomarkers for luminal A-like breast cancer. PloSOne 2014;9(1):87032.

12 Mitchell PS. Parkin RK. Kroh EM. Fritz BR. Wyman SK. Pogosova-Agadjanyan E L. Circulating microRNAs as stable blood-based markers for cancer detection. Proc Natl Acad Sci USA 2008105;(30):105138.

13. Brase JC. Johannes M. Schlomm T. Fälth M. Haese A. Steuber T. et al. Circulating miRNAs are correlated with tumo rprogression in prostate cancer. IntJCancer 2011; 128(3):60816

14. Bryant RJ, Pawlowski T, Catto JW, Marsden G, Vessella RL, Rhees B, et al. Changes in circulating micro RNA levels associated with prostate cancer. $\mathrm{Br}$ JCancer 2012; 106(4):76874.

15. Srivastava A. Goldberger H. Dimtchev A. Ramalinga M. Chijioke J. Marian C. et al .micro RNA profiling in prostate cancer-the diagnostic potential of urinary miR-205 and miR-214. PLoSOne 2013; 8(10):e76994.

16. Markou A. Liang Y. Lianidou E. Prognostic, therapeutic and diagnostic potential of microRNAs in non-small cell lung cancer. Clinical Chemistry and Laboratory Medicine 2011;49(10):15911603.

17. Zhu W. Liu X., He J. Chen D., Hunag Y. Zhang Y. K. Overexpression of members of the microRNA-183 family is a risk factor for lung cancer: a case control study. BMC Cancer2011;11:393.

18. Tang D. Shen Y. Wang M. et al. Identification of plasma microRNAs as novel noninvasive biomarkers for early detection of lung cancer. European Journal of Cancer Prevention 2013;22(6):540548.

19. Xing L. Todd N. W. Yu L. Fang H. Jiang F. Early detection of squamous cell lung cancer in sputum by a panel of microRNA markers. Modern Pathology 2010;23(8):11571164

20. Liu XG. Zhu WY. Huang YY. et al. High expression of serum miR-21 and tumor miR-200c associated with poor prognosis in patients with lung cancer. Medical Oncology. 2012;29(2):618-626.

21. Brase JC. Johannes M. Schlomm T. et al. Circulating miRNAs are correlated with tumor progression in prostate cancer. International Journal of Cancer 2011;128(3):608616.

22. Casanova-Salas I. Rubio-Briones J. Calatrava A. et al. Identification of miR-187 and miR-182 as Biomarkers of Early Diagnosis and Prognosis in Patients with Prostate Cancer Treated with Radical Prostatectomy. Journal of Urology 2014;1:107.

23. Maugeri-Saccà M. Coppola V. Bonci D. De Maria R. MicroRNAs and prostate cancer: from preclinical research to translational oncology. Cancer Journal 2012;18(3):253261.

24. Mahn R. Heukamp LC. Rogenhofer S. von Ruecker A. Muller SC. Ellinger J. Circulating microRNAs (miRNA) in serum of patients with prostate cancer. Urology 2011;77:9-16.

25. Yaman Agaoglu F. Kovancilar M. Dizdar Y. Darendeliler E. Holdenrieder S. Dalay N. Gezer U: Investigation of miR-21, miR-141, and miR-221 in blood circulation of patients with prostate cancer. 
Tumor Biol 2011;32:583-588.

26. Gonzales JC. Fink LM. Goodman OB Jr. Symanowski JT. Vogelzang NJ. Ward DC. Comparison of circulating MicroRNA 141 to circulating tumor cells, lactate dehydrogenase, and prostate-specific antigen for determining treatment response in patients with metastatic prostate cancer. Clin Genitourin Cancer 2011;9(1): 39-45.

27. Selth LA. Townley SL. Bert AG. Stricker PD. Sutherland PD. Horvath LG. Goodall GJ. Butler LM. Tilley WD. Circulating microRNAs predict biochemical recurrence in prostate cancer patients. $\mathrm{Br}$ JCancer 2013;109(3): 641-650.

28. Murakami Y. Yasuda T. Saigo K. et al. Comprehensive analysis of microRNA expression patterns in hepatocellular carcinoma and non-tumorous tissues. Oncogene 2006;25:25372545.

29 Ji J. Yamashita T. Budhu A. et al. Identification of microRNA-181 by genome-wide screening as a critical player in EpCAM-positive hepatic cancer stem cells. Hepatology2009;50:472480.

30. Budhu A. Jia HL. Forgues M. et al. Identification of metastasis-related microRNAs in hepatocellular carcinoma. Hepatology 2008;47:897907.

31. Ji J. Shi J. Budhu A. et al. MicroRNA expression, survival, and response to interferon in liver cancer. $N$ EnglJMed 2009;361:14371447.

32. Ladeiro Y. Couchy G. Balabaud C. et al. MicroRNA profiling in hepatocellular tumors is associated with clinical features and oncogene tumor suppressor gene mutations. Hepatology 2008;47:19551963

33. Parkin DM. Bray F. Ferlay J and Pisani P. Global cancer statistics, 2002. CA Cancer J Clin 2005; 55: 74108.

34. Guo Y. Chen Z. Zhang L. Shi S. Feng X. et al. Distinctive microRNA profiles relating to patient survival in esophageal squamous cell carcinoma. Cancer Res 2008; 68: 26-33.

35. Feber A. Xi L. Luketich JD. Pennathur A. Landreneau RJ. Wu M. et al. MicroRNA expression profiles of esophageal cancer. $J$ Thorac Cardiovasc Surg 2008; $135: 255-260$.

36. Mahdavinezhad A. Mousavi Bahar SH. Poorolajal J. Yadegarazari R. Jafari M. Shabab N. et al. Evaluation of miR-141, miR-200c, miR-30b Expression and
Clinicopathological Features of Bladder Cancer. International journal of molecular and cellular medicine 2015;4(1):32.

37. Zabdotneva AA, Zhavoronrov A, Garazha AV, Roumiantsev SA, Buzdin AA. Characteristics patterns of MicroRNA expression in huan bladder cancer. Frontiers in genetics, 2012:3

38. Ratert N. Meyer HA. Jung M. Lioudmer P. Mollenkopf HJ. Wagner I. et al. miRNA profiling identifies candidate mirnas for bladder cancer diagnosis and clinical outcome. The Journal of Molecular Diagnostics 2013;15(5):695705.

39. Kluiver J. Poppema S. de Jong D. Blokzijl T. Harms G. Jacobs S. Kroesen B.J. van den Berg A. BIC and miR155 are highly expressed in Hodgkin, primary mediastinal and diffuse large B cell lymphomas. $J$. Pathol 2005;207(2):243249.

40. Manfe V. Biskup E. Rosbjerg A. et al. miR-122 regulates p53/Akt signalling and the chemotherapyinduced apoptosis in cutaneous T-cell lymphoma. PLoSOne. 2012; 7( 1): e29541

41. Ballabio E. Mitchell T. van Kester MS. et al. MicroRNA expression in Sezary syndrome: identification, function, and diagnostic potential. Blood 2010;116(7): 110513.

42. Maj J. Jankowska-Konsur A. Sadakierska-Chudy A. et al. Altered microRNA expression in mycosis fungoides. Br J Dermatol2012; 166(2): 3316.

43. Yamanaka Y. Tagawa H. Takahashi N. et al. Aberrant overexpression of microRNAs activate AKT signaling via down-regulation of tumor suppressors in natural killer-cell lymphoma leukemia. Blood 2009; 114(15): 326575

44. Gottardo F. Liu CG. Ferracin M. et al. Micro-RNA profiling in kidney and bladder cancers. Urol Oncol.2007;25:387392.

45. Cheng T. Wang L. Li Y. Huang C. Zeng L. Yang J. Differential microRNA expression in renal cell carcinoma. Oncol Lett. 2013;6:769776.

46. Teixeira AL. Ferreira M. Silva J. et al. Higher circulating expression levels of miR-221 associated with poor overall survival in renal cell carcinoma patients. Tumour Biol. 2014;35:40574066.

47. Zhao J. Lei T. Xu C. et al. MicroRNA-187, downregulated in clear cell renal cell carcinoma and 
associated with lower survival, inhibits cell growth and migration though targeting B7-H3. Biochem Biophys Res Commun. 2013; 438:439444.

48. Wang J. He J. Su F. et al. Repression of ATR pathway by miR-185 enhances radiation-induced apoptosis and proliferation inhibition. Cell Death Dis. 2013; 4:e699.

49. S. Gilad. E. Meiri. Y. Yogev et al. Serum microRNAs arepromising novel biomarkers. PLoS One 2008; 3(9):e31-48.

50. K. Gocze. K. Gombos. K. Juhasz et al.Unique microRNA expression profiles in cervical cancer. Anticancer Research 2013; 33(6):2561-2567.

51. L. Huang. J.X. Lin. Y.H. Yu. M.Y. Zhang. H.-Y. Wang. And M. Zheng. Downregulation of six microRNAs is associated with advanced stage, lymph nodemetastasis and poor prognosis in small cell carcinoma of the cervix. PLoS One 2012;7(3): e33762.

52. Wilting SM. Verlaat W. Jaspers A. et al. Methylationmediated transcriptional repression of microRNAs during cervical carcinogenesis. Epigenetics 2013.8(2):220-228.

53. Sipos JA. Mazzaferri EL. Thyroid cancer epidemiology and prognostic variables. Clin Oncol ( $R$ Coll Radiol) 2010; 22: 395-404

54. He H. Jazdzewski K. Li W. Liyanarachchi S. Nagy R. Volinia S. et al. The role of microRNA genes in papillary thyroid carcinoma. Proc Natl Acad Sci USA. 2005;102: 19075-19080.

55. Zhang X. Li M. Zuo K. Li D. Ye M. Ding L. et al. Upregulated miR-155 in papillary thyroid carcinoma promotes tumor growth by targeting APC and activating Wnt/ $\beta$-catenin signaling. J Clin Endocrinol Metab. 2013; 98: E1305-1313.

56. Pallante P. Visone R. Ferracin M. Ferraro A. Berlingieri MT. Troncone G. et al. MicroRNA deregulation in human thyroid papillary carcinomas. Endocr Relat Cancer. 2006; 13:497-508.

57. Shen R. Liyanarachchi S. Li W. Wakely PE. Jr. Saji M. Huang J. et al. MicroRNA signature in thyroid fine needle aspiration cytology applied to "atypia of undetermined significance" cases. Thyroid 2012; 22: 9-16.
58. Yu S. Liu Y. Wang J. Guo Z. Zhang Q. Yu F. et al. Circulating microRNA profiles as potential biomarkers for diagnosis of papillary thyroid carcinoma. J Clin Endocrinol Metab. 2012; 97: 20842092.

59. Lee YS. Lim YS. Lee JC. Wang SG. Park HY. Kim SY. et al. Differential expression levels of plasma-derived miR-146b and miR-155 in papillary thyroid cancer. Oral Oncol. 2015; 51:77-83.

60. Suh KS. Park SW. Castro A. Patel H. Blake P. et al. Ovarian cancer biomarkers for molecular biosensors and translational medicine. Expert Rev Mol Diagn. 2010;10: 1069-1083.

61. Moss EL. Hollingworth J. Reynolds TM. The role of CA125 in clinical practice. J Clin Pathol 2005;58: 308-312.

62. Y.-W. Chung. HS. Bae. J.-Y. Song et al. Detection of microRNA as novel biomarkers of epithelial ovarian cancer from the serum of ovarian cancer patients. International Journal of Gynecological Cancer 2013; 23(4);673-679.

63. M. V. Iorio. R. Visone. G. Di Leva et al. MicroRNA signatures in human ovarian cancer. Cancer Research 2007;67(18);8699-8707.

64. E. Calura. R. Fruscio L. Paracchini et al. miRNA landscape in stage I epithelial ovarian cancer defines the histotype specificities. Clinical Cancer Research 2013;19(15);4114-4123.

65. K. Sawada. A. K. Mitra. A. R. Radjabi et al. Loss of Ecadherin promotes ovarian cancer metastasis via $\square 5$ integrin, which is a therapeutic target. Cancer Research 2008; 68(7): 2329-2339.

66. Lewis BP. Burge CB and Bartel DP. Conserved seed pairing, often flanked by adenosines, indicates that thousands of human genes are microRNA targets. Cell 2005.120: 15-20.

67. Ciafre SA. Galardi S. Mangiola A. Ferracin M. Liu CG. Sabatino G. Negrini M. Maira G. Croce CM and Farace MG. Extensive modulation of a set of microRNAs in primary glioblastoma. Biochem Biophys Res Commun 2005.334: 1351-1358.

68. Silber J, Lim DA.Petritsch C. Persson AI. Maunakea AK. Yu M. Vandenberg SR. Ginzinger DG. James CD. Costello JF. Bergers G. Weiss WA. Alvarez-Buylla A 
and Hodgson JG. miR-124 and miR-137 inhibit proliferation of glioblastoma multiforme cells and induce differentiation of brain tumor stem cells. $B M C$ Med 2008; 6: 14.

69. Zhi F, Chen X. Wang S. Xia X. Shi Y. Guan W. Shao N. Qu H. Yang C. Zhang Y. Wang Q. Wang R. Zen K. Zhang CY. Zhang J and Yang Y. The use of hsa-miR21, hsa-miR-181b and hsa-miR-106a as prognostic indicators of astrocytoma. Eur J Cancer 2010.46: 1640-1649.

70. Yang C. Wang C. Chen X. Chen S. Zhang Y. et al. Identification of seven serum micro RNAs from a genome-wide serum micro RNA expression profile as potential noninvasive biomarkers for malignant astrocytomas. Int J Cancer 2013. 132: 116-127.

71. Lages E, A Guttin. M El Atifi. Claire Ramus. Hélène Ipas MicroRNA and target protein patterns reveal physiopathological features of glioma subtypes. PLoS One 2011.

72. Costa FF. Bischof JM. Vanin EF. Lulla RR. Wang M, et al. Identification of microRNAs as potential prognostic markers in ependymoma. PLoS One 2011; 6: e25114.

73. MacDonald TJ, Brown KM, LaFleur B, Peterson K, Lawlor C, Chen Y, Packer RJ, Cogen P and Stephan DA: Expression profiling of medulloblastoma: PDGFRA and the RAS/MAPK pathway as therapeutic targets for metastatic disease. Nat Genet 2001.29: 143-152.

74. Mi S.Lu J. Sun M. et al. MicroRNA expression signaturesaccurately discriminate acute lymphoblastic leukemia from acute myeloid leukemia. Proc Natl Acad Sci USA 2007;104:19971-6.

75. Zhu YD.Wang L.Sun $C$ et al. Distinctive microRNAsignature is associated with the diagnosis and prognosis of acute leukemia. Med Oncol 2012.29:2323-31.

76. Wang F.Wang XS. Yang GH et al. miR-29a and miR142-3p downregulation and diagnostic implication in human acute myeloid leukemia. Mol Biol Rep 2012;39:2713-22.

77. Marton S. Garcia MR. Robello C et al. Small RNAs analysis in CLL reveals a deregulation of miRNAexpression and novel miRNA candidates of putative relevance in CLL pathogenesis. Leukemia 2008;22:330-8.

78. Babashah S, Sadeghizadeh M, Hajifathali A, et al.
Targetingof the signal transducer Smo links microRNA-326 to theoncogenic Hedgehog pathway in CD34+ CML stem/progenitor cells. Int J Cancer 2013.133:579-89.

79. Fulci V. Colombo T. Chiaretti $S$ et al. Characterizationof $\mathrm{B}$ - and $\mathrm{T}$-lineage acute lymphoblastic leukemia byintegrated analysis of MicroRNA and mRNA expression profiles. Genes Chromosomes Cancer 2009;48:1069-82.

80. S. Warnakulasuriya. Global epidemiology of oral and oropharyngealcancer. Oral Oncology 2009;45:309316.

81. K. Alibek A. Kakpenova. and Y. Baiken. Role of infectiousagents in the carcinogenesis of brain and head and neck cancers. Infectious Agents and Cancer 2013;8:7

82. S. Kimura. S. Naganuma. D. Susuki et al. Expression of micro-RNAs in squamous cell carcinoma of human head and neck and the esophagus: MiR-205 and miR21 are specific markers forHNSCC and ESCC. Oncology Reports 2010;23:1625-1633.

83. M. Avissar. M. D. McClean. K. T. Kelsey. and C. J. Marsit. MicroRNA expression in head and neck cancer associates withalcohol consumption and survival. Carcinogenesis 2009;30:2059-2063.

84. G. Childs. M. Fazzari.G. Kung et al. Low-level expressionof microRNAs let-7d and miR-205 are prognostic markers ofhead and neck squamous cell carcinoma. American Journal of Pathology 2009; 174:736-745.

85. A. B. Y. Hui. M. Lenarduzzi. T. Krushel et al. ComprehensiveMicroRNA profiling for head and neck squamous cell carcinomas. Clinical Cancer Research 2010;16:1129-1139.

86. N. J. Park. H. Zhou. D. Elashoff et al. Salivary microRNA:discovery, characterization, and clinical utility for oral cancerdetection. Clinical Cancer Research 2009;15(17):5473-5477.

87. C.-J. Liu, S.-C. Lin, C.-C. Yang, H.-W. Cheng and K.W. Chang. Exploiting salivary miR-31 as a clinical biomarker of oral squamous cell carcinoma. Head and Neck2012;34(2):219-224.

88. T.S. Wong. X.-B. Liu B. Y.-H. Wong. R. W.-M. Ng, A. P.-W.Yuen. and W. I. Wei. Mature miR-184 as potential oncogenicmicroRNA of squamous cell carcinoma of tongue. ClinicalCancer Research 2008;14(9):2588-2592. 